\title{
On the Connectivity of Boundaries of Sets Minimizing Perimeter Subject to a Volume Constraint
}

\author{
Peter Sternberg ${ }^{1}$ And Kevin Zumbrun ${ }^{2}$
}

We consider the problem of locally minimizing perimeter within a given bounded domain $\Omega \subset \mathbb{R}^{n}$ subject to a volume constraint. By a local minimizer, we mean a set of finite perimeter $E \subset \Omega$ satisfying the condition

$$
P(E, \Omega) \leq P(F, \Omega)
$$

among all competitors $F \subset \Omega$ such that $|F|=|E|$ and such that $\left\|\chi_{F}-\chi_{E}\right\|_{L^{1}(\Omega)}<\delta$ for some $\delta>0$. We prove that when $\Omega$ is convex, the boundary $\partial E \cap \Omega$ is connected, or else $\partial E \cap \Omega$ consists of parallel planes meeting $\partial \Omega$ orthogonally. The result arises as an application of a property we derive for normal variations of constant mean curvature hypersurfaces bounding sets within a convex domain $\Omega$. The property states that for such variations, area is a concave function of the enclosed volume. Our results hold in all dimensions $n$, even in the presence of singularities.

\section{Introduction.}

We consider the problem of locally minimizing perimeter within a given bounded domain $\Omega \subset \mathbb{R}^{n}$ subject to a volume constraint. By a local minimizer, we mean a set of finite perimeter $E \subset \Omega$ satisfying the condition

$$
P(E, \Omega) \leq P(F, \Omega)
$$

\footnotetext{
${ }^{1}$ Research supported in part by the N.S.F. under grant number DMS-9322617 and a U.S.-Israel Bi-national Grant.

${ }^{2}$ Research supported in part by the O.N.R. under grant number N00014-94-10456 and the N.S.F. under grant number DMS-9107990.
} 
among all competitors $F$ such that the measure of $F$ equals the measure of $E$ and such that the characteristic function of $F$ lies within some $L^{1}(\Omega)$ neighborhood of the characteristic function of $E$. Here $P(\cdot, \Omega)$ refers to perimeter within $\Omega$. A precise definition of perimeter is given at the beginning of Section 2; we refer to [G] for further background.

The regularity of solutions to this problem was studied in arbitrary smooth domains $\Omega$ during the 1980's in [GMT] and [Gr]. There it was shown that the boundary of any solution $E$ is analytic in $\Omega$ off of a singular set of small Hausdorff dimension and that the boundary is of constant mean curvature. (See Theorem 2.1 for a precise statement.) Thus, in the plane for example, a typical solution might have boundary consisting of a number of circular arcs meeting $\partial \Omega$ normally (since orthogonality arises as a natural boundary condition). In higher dimensions, of course, the condition of constant mean curvature could yield much more complicated hypersurfaces.

We show here, however, that for $\Omega$ convex, the structure of local minimizers is relatively simple, regardless of dimension; namely,

The boundary of a local minimizer $E$ must be connected

(cf. Theorem 2.6). The only exception occurs when $\Omega$ is locally a cylinder, in which case local minimizers can arise with $\partial E \cap \Omega$ consisting of a union of parallel planar components whose normals coincide with the axial direction of the cylinder. We note that when $\Omega$ is nonconvex, one can easily construct solutions-even absolute minimizers- with multiple boundary components.

Our connectivity result arises as an application of a property we derive for normal variations of constant mean curvature hypersurfaces bounding sets within convex domains. Roughly, this property states that, provided the singular set is of sufficiently low dimension,

Under a normal variation, the perimeter of a constant mean curvature hypersurface is a concave function of the volume it bounds

(cf. Theorem 2.5).

To illustrate this principle and to see in a heuristic way how it leads to a connectivity result, consider the simpler problem of minimizing perimeter in $\Omega=\mathbb{R}^{n}$ subject to a volume constraint $m$. By the Isoperimetric Theorem, any local minimizer $E$ would have to consist of a collection of balls $B_{i}$ of radius $r_{i}$, perimeter $P_{i}=n \omega_{n} r_{i}^{n-1}$ and volume $V_{i}=\omega_{n} r_{i}^{n}$ with $\sum_{i} V_{i}=m$. Then the perimeter of each ball is seen to be a concave function of its volume:

$$
P_{i}\left(V_{i}\right)=C V_{i}^{\frac{n-1}{n}}
$$


Now suppose, for example, a local minimizer $E$ consists of two such balls so that $V_{1}+V_{2}=m$ and so the total perimeter $P=P_{1}+P_{2}$ can be expressed as a function of $V_{1}$ alone as

$$
P\left(V_{1}\right)=C\left(V_{1}^{\frac{n-1}{n}}+\left(m-V_{1}\right)^{\frac{n-1}{n}}\right) .
$$

By stationarity, $P^{\prime}\left(V_{1}\right)=0$, leading to the requirement that the two balls must have the same mean curvature (or equivalently, the same radius). However, the concavity of the perimeter/volume relationship implies that $P^{\prime \prime}\left(V_{1}\right)<0$, contradicting the local minimality of the configuration $E$. Thus, $E$ must consist of only one ball.

In the case of a bounded domain $\Omega$, local minimizers $E$ will not necessarily have spherical boundaries and indeed, as was mentioned earlier, $\partial E$ may possess singularities. Matters are also complicated by the need to consider the behavior of the free surface near $\partial \Omega$. Nonetheless, the argument is in spirit quite analogous to the one just presented. We should mention that the concavity property derived here is reminiscent of a more familiar fact regarding normal variations of minimal surfaces. If $M$ is a hypersurface with mean curvature zero and normal vector $\nu_{M}$ and we consider normal variations $M_{t}=\left\{x+t \zeta(x) \nu_{M}(x): x \in M\right\}$, then a standard calculation yields

$$
\left.\frac{d^{2}\left(\text { Area of } M_{t}\right)}{d t^{2}}\right|_{t=0}=\int_{M}\left|\nabla_{M} \zeta\right|^{2}-\zeta^{2}\left\|B_{M}\right\|^{2} d H^{n-1}(x)
$$

where $\nabla_{M} \zeta$ denotes the gradient relative to $M$ of a compactly supported scalar function $\zeta, B_{M}$ denotes the second fundamental form associated with $M$ and $\left\|B_{M}\right\|^{2}=\sum_{i=1}^{n-1} \kappa_{i}^{2}$ where $\left\{\kappa_{i}\right\}$ are the principal curvatures (cf. [S], Section 9). The difference between our result and (1.2) is not just that we have constant rather than zero mean curvature, but also that the second derivative of area is taken with respect to volume, not $t$. Indeed, for constant mean curvature hypersurfaces, the second $t$-derivative will not in general be negative. In the case of a ball in $\mathbb{R}^{n}$ of radius $t$, for example, the relationship $P(t)=C t^{n-1}$ is convex, not concave. (For the analogue of (1.2) for constant mean curvature hypersurfaces in bounded domains, see formula (2.46).)

In addition to its purely geometric interest, our work is motivated by the connection which exists between the volume-constrained least area problem (1.1) and a well-studied variational problem related to phase transitions:

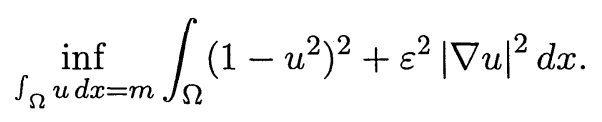


Here $u: \Omega \rightarrow \mathbb{R}^{1}$ is viewed as an order parameter, and a minimizer $u_{\varepsilon}$ is forced by the integral constraint to make a rapid transition from one phase $\left\{u_{\varepsilon} \approx 1\right\}$ to another $\left\{u_{\varepsilon} \approx-1\right\}$. It can be shown that for small values of $\varepsilon$, local minimizers of (1.3) are closely related to local minimizers of (1.1) (cf. [KS]). Our connectivity result here is analogous to a similar result restricting the structure of transitions for stable critical points of (1.3) when $\Omega$ is strictly convex (see [SZ] for this result as well as a list of references for (1.3)). Indeed, the arguments here inspired the proofs in the continuum setting of (1.3).

We conclude this paper with another application of the concavity of the perimeter/volume relationship for normal variations. It states that for absolute minimizers of (1.1) in convex domains, the perimeter function

$$
P(m) \equiv \inf P(F, \Omega) \quad \text { taken over }\{F \subset \Omega: \text { measure of } F=m\}
$$

is a concave function volume $m$ (cf. Theorem 2.8). Since $P$ is symmetric about $|\Omega| / 2$, this has the interesting consequence that for $m<|\Omega| / 2$, the mean curvature of an absolute minimizer must be strictly positive (cf. Corollary 2.9). We remark that this is not generally true for local minimizers.

\section{Main Results.}

Notation and Preliminaries: We denote by $|E|$ the $n$-dimensional Lebesgue measure of a set $E \subset \mathbb{R}^{n}$ and by $H^{\alpha}(\cdot), \alpha$-dimensional Hausdorff measure. We use $\bar{E}$ to denote the topological closure of a set $E$. Balls of radius $r$ and center $x$ in $\mathbb{R}^{n}$ are denoted by $B_{r}(x)$. For any open set $\Omega \subset \mathbb{R}^{n}$ we recall that a function $f \in L^{1}(\Omega)$ is of bounded variation in $\Omega$ if

$$
\|\nabla f\|(\Omega) \equiv \sup \left\{\int_{\Omega} f \operatorname{div} g d x: g \in C_{0}^{1}\left(\Omega ; \mathbb{R}^{n}\right),|g| \leq 1\right\}<\infty .
$$

A set $E \subset \mathbb{R}^{n}$ is said to be of finite perimeter in $\Omega$ if $\chi_{E}$ is of bounded variation in $\Omega$ (cf. [G]), where $\chi_{E}$ denotes the characteristic function of the set $E$.

For an open, bounded set $\Omega \subset \mathbb{R}^{n}$, we will consider the perimeter functional $E \rightarrow P(E, \Omega)$ where

$$
P(E, \Omega)=\left\|\nabla \chi_{E}\right\|(\Omega) .
$$

Since the perimeter is not changed by sets of measure zero we may, for convenience, always assume that

$$
0<\left|E \cap B_{r}(x)\right|<\omega_{n} r^{n}
$$


for every ball $B_{r}(x)$ such that $x \in \partial E$, where $\omega_{n} \equiv\left|B_{1}(x)\right|$. If, for instance, $E$ has $C^{2}$ boundary, then $P(E, \Omega)=H^{n-1}(\partial E \cap \Omega)$.

We now set

$$
\mathcal{A}_{m}=\left\{E \subset \Omega: \chi_{E} \in B V(\Omega),|E|=m\right\} \text { for any } m \in[0,|\Omega|] .
$$

Then by a local minimizer of perimeter subject to fixed volume, we mean a set $E \in \mathcal{A}_{m}$ such that

$$
P(E, \Omega) \leq P(F, \Omega) \quad \text { whenever } F \in \mathcal{A}_{m} \text { and }\left\|\chi_{E}-\chi_{F}\right\|_{L^{1}(\Omega)}<\delta
$$

for some positive $\delta$.

The regularity theory for solutions to (2.1) has been well-studied and in the theorem below, we combine the known interior and boundary regularity results.

Theorem 2.1. (cf. [GMT]), [Gr]. Let $\Omega$ be an open, bounded connected set in $\mathbb{R}^{n}$ with $C^{2, \alpha}$ boundary, and let $E$ be a local minimizer of perimeter with respect to fixed volume in the sense of (2.1). Then there exists a closed subset of $\overline{\Omega \cap \partial E}$ denoted by sing $\overline{\Omega \cap \partial E}$ such that $\operatorname{sing} \overline{\Omega \cap \partial E}=\emptyset$ if $n \leq 7$ while $H^{n-8+\gamma}(\operatorname{sing} \overline{\Omega \cap \partial E})=0$ for all $\gamma>0$ if $n \geq 8$. On the complement of this singular set, reg $\overline{\Omega \cap \partial E} \equiv \overline{\Omega \cap \partial E} \backslash$ sing $\overline{\Omega \cap \partial E}$, we have:

(i) There is a constant $H$ such that in a neighborhood of every $x \in$ (reg $\overline{\Omega \cap \partial E}) \cap \Omega, \partial E$ is real analytic and of constant mean curvature $H$.

(ii) For every $x \in(\operatorname{reg} \overline{\Omega \cap \partial E}) \cap \partial \Omega$, the set $\overline{\Omega \cap \partial E}$ can be represented locally as the graph of a $C^{2, \alpha}$ function which meets $\partial \Omega$ orthogonally.

Remark. In light of the low Hausdorff dimension of the singular set, we need not make a distinction between integrals

$$
\int_{\Omega \cap \partial E} \cdot d H^{n-1}(x)
$$

and

$$
\int_{\Omega \cap \operatorname{reg} \partial E} \cdot d H^{n-1}(x)
$$

or between

$$
\int_{\partial \Omega \cap(\overline{\Omega \cap \partial E})} \cdot d H^{n-2}(x)
$$


and

$$
\int_{\partial \Omega \cap \operatorname{reg}(\overline{\Omega \cap \partial E})} \cdot d H^{n-2}(x)
$$

We also note that this estimate on the singular set is optimal, as can be shown by explicit example.

Proof. The estimate on the Hausdorff dimension of the singular set in the interior, as well as the interior regularity and constant mean curvature property (i) follow from [GMT]. In their work, as well as that of [Gr], the results are stated for absolute minimizers rather than local ones but the proofs in both papers carry over without change to the local minimizer setting. The estimate on the singular set at the boundary, as well as $C^{1, \alpha}$ boundary regularity and orthogonality (ii) follow from [G]. Then $C^{2, \alpha}$ regularity follows from standard Schauder theory. We should mention that while the boundary regularity result in $[\mathrm{Gr}]$ is stated as $C^{1}$ regularity, a closer inspection of the proof reveals that it is in fact $C^{1, \alpha}$ regularity which has been established.

Now let $M$ be any connected component of $\overline{\Omega \cap \partial E}$, where $E$ is a local minimizer of perimeter with respect to fixed volume in the sense of (2.1). Take $\zeta \in C^{\infty}\left(\mathbb{R}^{n}\right)$ to be any non-negative function vanishing in a neighborhood of sing $M$. If sing $M=\emptyset$, take $\zeta \equiv 1$. We will associate with any such $\zeta$ a family of diffeomorphisms $\left\{\Psi_{t}\right\}$ of $\mathbb{R}^{n}$ in the following way. First let $Y \in C^{2}\left(\mathbb{R}^{n} ; \mathbb{R}^{n}\right)$ be a vector field satisfying:

$$
\begin{array}{ll}
\text { spt } Y \subset \mathcal{O}, & \text { where } \mathcal{O} \subset \mathbb{R}^{n} \text { is open, } \\
& \text { and }(\overline{\Omega \cap \partial E}) \cap \mathcal{O}=M, \\
|Y(x)|=1 \quad & \begin{array}{l}
\text { for all } x \in \mathbb{R}^{n} \text { lying in some } \\
\text { neighborhood of } M-\{\zeta=0\}
\end{array} \\
Y(x) \in T_{x}(\partial \Omega) & \text { for all } x \in \partial \Omega \\
Y(x)=\nu_{M}(x) & \text { for all } x \in M-\{\zeta=0\} .
\end{array}
$$

Here $\nu_{M}$ denotes the outer unit normal to $M$ with respect to the set $E$ and $T_{x}(\partial \Omega)$ denotes the tangent space to the manifold $\partial \Omega$ at $x$. Note that the construction of such a smooth vector field $Y$ is feasible in light of Theorem 2.1. Now define the vector field $X \in C^{2}\left(\mathbb{R}^{n} ; \mathbb{R}^{n}\right)$ by the formula

$$
X=\zeta Y
$$


and introduce the family of diffeomorphisms $\left\{\Psi_{t}\right\}$ (for small $|t|$ ) as the solution to:

$$
\frac{\partial \Psi_{t}(x)}{\partial t}=X\left(\Psi_{t}(x)\right), \quad \Psi_{0}(x)=x \quad \text { for all } x \in \mathbb{R}^{n} .
$$

Note that $\Psi_{t}=I$ on $\mathbb{R}^{n} \backslash \mathcal{O}$ and by (2.4) it follows that $\Psi_{t}$ maps $\partial \Omega$ into $\partial \Omega$. Therefore, in particular, $E_{t} \equiv \Psi_{t}(E) \subset \Omega$. Note also that in the absence of singularities, $\Psi_{t}(x) \sim x+t \nu_{M}(x)$ for $x \in M$. Now we define $A(t)=\mathrm{P}\left(E_{t}, \Omega\right)$ and $V(t)=\left|E_{t}\right|$. From the properties of $Y$ and $\zeta$ one sees that $V$ is an increasing function of $t$. Hence, we may view the perimeter of $E_{t}$ within $\Omega$ as a function of its volume $V$. We denote this function by $\mathcal{A}(V)$ and now establish the familiar relationship between area and volume:

Proposition 2.2. Let $\Omega \subset \mathbb{R}^{n}$ be any bounded open domain with $C^{2, \alpha}$ boundary. Let $M$ be any connected component of $\overline{\Omega \cap \partial E}$ where $E$ is a local minimizer of perimeter with respect to fixed volume in the sense of (2.1), and denote the (constant) mean curvature of reg $M$ by $H$. For any smooth, non-negative function $\zeta: \mathbb{R}^{n} \rightarrow \mathbb{R}^{1}$ vanishing in a neighborhood of sing $M$ with spt $\zeta \cap M \cap \Omega \neq \emptyset$, let $Y$ be a vector field satisfying (2.2)-(2.5), let $X=\zeta Y$ and let $\left\{\Psi_{t}\right\}$ be a family of diffeomorhisms satisfying (2.7). Then the function $\mathcal{A}(V)$ describing the perimeter of the family of sets $E_{t} \equiv \Psi_{t}(E)$ as a function of their volume satisfies the relation:

$$
\left.\frac{d \mathcal{A}}{d V}\right|_{V=|E|}=(n-1) H
$$

Proof. The formula will follow from the chain rule once we compute the quantities $\left.\frac{d V}{d t}\right|_{t=0}$ and $\left.\frac{d A}{d t}\right|_{t=0}$. We begin with the first derivative of volume. Denote by $J \Psi_{t}(x)$ the Jacobian of the mapping $\Psi_{t}: \mathbb{R}^{n} \rightarrow \mathbb{R}^{n}$ evaluated at $x$. Then we have

$$
\left.\frac{d V}{d t}\right|_{t=0}=\left.\frac{d}{d t}\right|_{t=0} \int_{\Psi_{t}(E)} 1 d x=\left.\int_{E} \frac{\partial J \Psi_{t}(x)}{\partial t}\right|_{t=0} d x .
$$

Recalling (2.7), we can write

$$
J \Psi_{t}=\operatorname{det}\left(I+t \nabla X+\frac{1}{2} t^{2} \nabla Z+o\left(t^{2}\right)\right)
$$

where $\nabla$ denotes the gradient with respect to $x$ and $\left.Z \equiv \frac{\partial^{2} \Psi_{t}}{\partial t^{2}}\right|_{t=0}$ has $i^{\text {th }}$ component given by

$$
Z^{(i)}=X_{x_{j}}^{(i)} X^{(j)}
$$


Here and throughout we invoke the summation convention for repeated indices and the subscript denotes differentiation with respect to $x_{j}$. Utilizing the general matrix identity

$$
\begin{aligned}
\operatorname{det}(I+t A+ & \left.\frac{1}{2} t^{2} B+o\left(t^{2}\right)\right)=1+t \operatorname{trace} A \\
& +\frac{1}{2} t^{2}\left[\operatorname{trace} B+(\operatorname{trace} A)^{2}-\operatorname{trace}\left(A^{2}\right)\right]+o\left(t^{2}\right)
\end{aligned}
$$

we find

$$
\left.\frac{\partial J \Psi_{t}}{\partial t}\right|_{t=0}=\operatorname{div} X .
$$

Hence we can use the Divergence Theorem to compute

$$
\begin{aligned}
\left.\frac{d V}{d t}\right|_{t=0}= & \int_{E} \operatorname{div} X d x=\int_{M} X \cdot \nu_{M} d H^{n-1}(x) \\
& +\int_{(\partial E \backslash M) \cap \Omega} X \cdot \nu_{\partial E} d H^{n-1}(x)+\int_{\partial E \cap \partial \Omega} X \cdot \nu_{\partial \Omega} d H^{n-1}(x) .
\end{aligned}
$$

Here $\nu_{\partial E}$ and $\nu_{\partial \Omega}$ denote the outer unit normals to $\partial E$ and $\partial \Omega$ respectively. Recall that $X=\zeta Y$ where the vector field $Y$ satisfies (2.2)-(2.5). Hence, the last two boundary integrals vanish and we obtain

$$
\left.\frac{d V}{d t}\right|_{t=0}=\int_{M} \zeta d H^{n-1}(x) .
$$

(Note that in the absence of singularities, $\zeta \equiv 1$ so that the $t$-derivative of volume is simply area.)

We now turn to the calculation of $\left.\frac{d A}{d t}\right|_{t=0}$. The computation is similar to the one above with the role of $\Psi_{t}$ replaced by the mapping

$$
\Phi_{t} \equiv\left(\Psi_{t}\right)_{\left.\right|_{M}}: M \rightarrow \bar{\Omega} .
$$

We find

$$
\left.\frac{d A}{d t}\right|_{t=0}=\left.\int_{M} \frac{\partial J \Phi_{t}(x)}{\partial t}\right|_{t=0} d x
$$

where $J \Phi_{t}$ denotes the Jacobian of the map $\Phi_{t}$. If one introduces

$$
\left\{\tau_{i}(x)\right\}_{i=1, \ldots, n-1}
$$


On the connectivity of boundaries of sets of minimizing perimeter 207

as an orthonormal basis for $T_{x} M$ for each $x \in$ reg $M$, then a brief calculation (and another appeal to (2.12)) yields

$$
\begin{array}{r}
J \Phi_{t}=1+t \operatorname{div}_{M} X+\frac{1}{2} t^{2}\left(\operatorname{div}_{M} Z+\left(\operatorname{div}_{M} X\right)^{2}+\sum_{i=1}^{n-1}\left|\left(D_{\tau_{i}} X\right)^{\perp}\right|^{2}\right. \\
\left.-\sum_{i, j=1}^{n-1}\left(\tau_{i} \cdot D_{\tau_{j}} X\right)\left(\tau_{j} \cdot D_{\tau_{i}} X\right)\right)+o\left(t^{2}\right) .
\end{array}
$$

We refer the reader to $[\mathrm{S}]$, Section 9 for details. (See also [G], Chapter 10.) Here $D_{\tau_{i}}$ denotes the directional derivative in the direction $\tau_{i}, \operatorname{div}_{M}(\cdot)$ denotes the divergence relative to the manifold $M$ given by

$$
\operatorname{div}_{M}(\cdot)=\left(D_{\tau_{i}}(\cdot)\right) \cdot \tau_{i},
$$

and $(\cdot)^{\perp}$ denotes orthogonal projection in the direction of $\nu_{M}$. Using (2.15) and (2.16) we then obtain

$$
\left.\frac{d A}{d t}\right|_{t=0}=\int_{M} \operatorname{div}_{M} X d H^{n-1}(x) .
$$

Since

$$
\begin{aligned}
\operatorname{div}_{M} X & =D_{\tau_{i}}\left(\zeta \nu_{M}\right) \cdot \tau_{i} \\
& =\left(D_{\tau_{i}} \zeta\right)\left(\nu_{M} \cdot \tau_{i}\right)+\zeta\left(D_{\tau_{i}} \nu_{M}\right) \cdot \tau_{i} \\
& =(n-1) H \zeta,
\end{aligned}
$$

formula (2.17) yields

$$
\left.\frac{d A}{d t}\right|_{t=0}=(n-1) H \int_{M} \zeta d H^{n-1}(x) .
$$

Together, (2.19) and (2.14) yield (2.8).

Crucial to our purpose is a formula for the second derivative of the function $\mathcal{A}(V)$ as well. For this, we will need the following proposition and lemma.

Proposition 2.3. Let $\Omega$ be an open, bounded, connected set in $\mathbb{R}^{n}$ with $C^{2, \alpha}$ boundary, and let $E$ be a local minimizer of perimeter with respect to fixed 
volume in the sense of (2.1). Then there exist positive constants $\mu$ and $C_{0}$ depending on $\partial \Omega, \delta$ and $E$ such that for all positive numbers $r<\mu$ we have

$H^{n-1}\left[(\overline{\Omega \cap \partial E}) \cap B_{r}\right] \leq C_{0} r^{n-1}$ for all balls $B_{r} \subset \mathbb{R}^{n}$ of radius $r$.

Proof. In [GMT] and [Gr], it is shown that $E$ is 'almost minimizing' without regard to the volume constraint in the following sense: there are positive numbers $\mu$ and $k$ such that for every ball $B_{r}, r<\mu$, and every set $F \subset \Omega$ of finite perimeter satisfying the condition

$$
F \backslash B_{r}=E \backslash B_{r},
$$

we have

$$
P(E, \Omega) \leq P(F, \Omega)+k r^{n} .
$$

Take $F$ in (2.22) to minimize perimeter within $B_{r}$ subject to condition (2.21). The monotonicity formula for area-minimizing sets applied to $\overline{\Omega \cap \partial F}$ within $B_{r}$ yields a uniform bound on the ratio

$$
\frac{H^{n-1}\left[(\overline{\Omega \cap \partial F}) \cap B_{r}\right]}{r^{n-1}}
$$

for all balls $B_{r}$ of sufficiently small radius. For balls $B_{r} \subset \Omega$, the monotonicity formula is standard (see e.g. [S], Section 17), while for balls intersecting $\partial \Omega$, a monotonicity formula yielding a uniform bound on the ratio (2.23) was established in [GJ], Section 3. The result for $E$ follows from (2.22) and the uniform bound on the ratio (2.23).

Lemma 2.4. Let $\Omega \subset \mathbb{R}^{n}$ be an open, bounded connected set with $C^{2, \alpha}$ boundary. Let $M$ be any connected component of $\overline{\Omega \cap \partial E}$, where $E$ is a local minimizer of perimeter with respect to fixed volume in the sense of (2.1). Then for any positive $\varepsilon>0$, there exist open sets $U^{\prime} \subset \subset U \subset \mathbb{R}^{n}$ with sing $M \subset U^{\prime}$ and $U \subset\{x: \operatorname{dist}(x, \operatorname{sing} M)<\varepsilon\}$ and there exists a function $\zeta \in C^{\infty}\left(\mathbb{R}^{n}\right)$ such that $0 \leq \zeta(x) \leq 1$ with

$$
\zeta(x)= \begin{cases}0 & \text { for } x \in U^{\prime} \\ 1 & \text { for } x \in \mathbb{R}^{n}-U\end{cases}
$$

and

$$
\int_{M}\left|\nabla_{M} \zeta(x)\right|^{2} d H^{n-1}(x) \leq C \varepsilon
$$


On the connectivity of boundaries of sets of minimizing perimeter 209

for some positive constant $C=C(\partial \Omega, \delta, E, n)$ independent of $\varepsilon$. Here $\nabla_{M}$ denotes the gradient relative to $M$.

Proof. Let $\varepsilon>0$ be given. From Theorem 2.1 we have, in particular, that $H^{n-3}(\operatorname{sing} M)=0$ so we may cover sing $M$ with balls $B_{r_{k}}\left(z_{k}\right)$ where $z_{k} \in \mathbb{R}^{n}$ and

$$
\sum_{k} r_{k}^{n-3}<\varepsilon
$$

Furthermore, we may require $r_{k}<\min \{\varepsilon / 2, \mu / 2\}$ where $\mu$ is the parameter in the statement of Proposition 2.3. We may also suppose this collection of balls is finite since sing $M$ is compact. Now for each $k$, let $\zeta_{k} \in C^{\infty}\left(\mathbb{R}^{n}\right)$ satisfy $0 \leq \zeta_{k} \leq 1$ with

$$
\zeta_{k}(x)= \begin{cases}0 & \text { for } x \in B_{r_{k}}\left(z_{k}\right) \\ 1 & \text { for } x \in \mathbb{R}^{n} \backslash B_{2 r_{k}}\left(z_{k}\right)\end{cases}
$$

and

$$
\left|\nabla \zeta_{k}(x)\right| \leq \frac{2}{r_{k}} \text { for all } x
$$

Then define $\tilde{\zeta}$ through the formula $\tilde{\zeta}(x)=\min _{k} \zeta_{k}(x)$. It follows that $0 \leq$ $\tilde{\zeta} \leq 1$ with

$$
\tilde{\zeta}=0 \quad \text { on } \quad U^{\prime} \equiv \cup_{k} B_{r_{k}}\left(z_{k}\right) \supset \operatorname{sing} M
$$

while

(2.29) $\tilde{\zeta}=1$ on $\mathbb{R}^{n} \backslash U$ where

$$
U \equiv \cup_{k} B_{2 r_{k}}\left(z_{k}\right) \subset\{x: \operatorname{dist}(x, \operatorname{sing} M)<\varepsilon\} .
$$


Furthermore, $\tilde{\zeta}$ is piecewise smooth and in light of Proposition 2.3, (2.26) and (2.27), we have

$$
\begin{aligned}
\int_{M}\left|\nabla_{M} \tilde{\zeta}(x)\right|^{2} d H^{n-1}(x) & \leq \int_{M}|\nabla \tilde{\zeta}(x)|^{2} d H^{n-1}(x) \\
& \leq \int_{M} \sum_{k}\left|\nabla \zeta_{k}(x)\right|^{2} d H^{n-1}(x) \\
& =\sum_{k} \int_{\left(B_{2 r_{k}}\left(z_{k}\right)-B_{r_{k}}\left(z_{k}\right)\right) \cap M}\left|\nabla \zeta_{k}(x)\right|^{2} d H^{n-1}(x) \\
& \leq \sum_{k} \frac{4}{r_{k}^{2}} H^{n-1}\left[B_{2 r_{k}}\left(z_{k}\right) \cap M\right] \\
& \leq 2^{n+1} C_{0} \sum_{k} r_{k}^{n-3}<C \varepsilon .
\end{aligned}
$$

Finally, we mollify $\tilde{\zeta}$ to obtain a $C^{\infty}$ function $\zeta$ still satisfying an estimate like (2.30). (As $\zeta$ is to satisfy (2.24), the mollification will slightly alter the definition of $U^{\prime}$ and $U$ from (2.28) and (2.29) above.)

We can now obtain the second derivative of perimeter with respect to volume.

Theorem 2.5. Let $\Omega \subset \mathbb{R}^{n}$ be any bounded, open, connected set with $C^{2, \alpha}$ boundary. Let $M$ be any connected component of $\overline{\Omega \cap \partial E}$ where $E$ is a local minimizer of perimeter with respect to fixed volume in the sense of (2.1). Then there exists a smooth non-negative function $\zeta: \mathbb{R}^{n} \rightarrow \mathbb{R}^{1}$ with spt $\zeta \cap M \cap \Omega \neq \emptyset$ and $\zeta$ vanishing in a neighborhood of sing $M$, there exists a vector field $Y$ satisfying (2.2)-(2.5), a vector field $X=\zeta Y$ and there exists a family of diffeomorphisms $\left\{\Psi_{t}\right\}$ satisfying (2.7), such that the function $\mathcal{A}(V)$ describing the perimeter of the family of sets $E_{t} \equiv \Psi_{t}(E)$ as a function of their volume satisfies the relation:

$$
\begin{aligned}
\left.\frac{d^{2} \mathcal{A}}{d V^{2}}\right|_{V=|E|}= & -\left(\frac{\int_{M} \zeta^{2}\left\|B_{M}\right\|^{2} d H^{n-1}(x)+\int_{M \cap \partial \Omega} \zeta^{2} B_{\partial \Omega}\left(\nu_{M}, \nu_{M}\right) d H^{n-2}(x)}{\left(\int_{M} \zeta d H^{n-1}(x)\right)^{2}}\right) \\
& +\frac{\int_{M}\left|\nabla_{M} \zeta\right|^{2} d H^{n-1}(x)}{\left(\int_{M} \zeta d H^{n-1}(x)\right)^{2}} .
\end{aligned}
$$

Here $B_{M}$ and $B_{\partial \Omega}$ denote the second fundamental form associated with $M$ and $\partial \Omega$ respectively.

Furthermore, if $\Omega$ is convex, then we have

$$
\left.\frac{d^{2} \mathcal{A}}{d V^{2}}\right|_{V=|E|}<0
$$


unless $M$ is planar $\left(\left\|B_{M}\right\| \equiv 0\right)$ and $B_{\partial \Omega}\left(\nu_{M}, \nu_{M}\right)=0$ on $M \cap \partial \Omega$, in which case

$$
\left.\frac{d^{2} \mathcal{A}}{d V^{2}}\right|_{V=|E|}=0
$$

Remark. In the absence of singularities such as in the case $n<8$, one can choose $\zeta \equiv 1$ and formula (2.31) simplifies to read:

$$
\left.\frac{d^{2} \mathcal{A}}{d V^{2}}\right|_{V=|E|}=-\left(\frac{\int_{M}\left\|B_{M}\right\|^{2} d H^{n-1}(x)+\int_{M \cap \partial \Omega} B_{\partial \Omega}\left(\nu_{M}, \nu_{M}\right) d H^{n-2}(x)}{H^{n-1}(M)^{2}}\right)
$$

Remark. In case $\Omega$ is strictly convex in the sense that

$$
B_{\partial \Omega}(\tau(x), \tau(x))>0 \quad \text { for every } x \in \partial \Omega \quad \text { and } \tau(x) \in T_{x}(\partial \Omega),
$$

then (2.32) will hold even if $M$ is planar.

Proof. If $\left\|B_{M}\right\| \equiv 0$ on reg $M$, every component of reg $M$ must lie in a plane. It then follows that there can be only one component of reg $M$ and that sing $M=\emptyset$ and we may choose $\zeta \equiv 1$ in the construction of the vector field $X$. To see this, note that one could blow up $E$ about any point in sing $M \cap \Omega$ to obtain a minimal cone $C$ (cf. [G]) with reg $(\partial C)$ consisting of components lying on hyperplanes. If $C$ were not a half-space, then necessarily the dimension of $\operatorname{sing}(\partial C)$ would be $n-2$ from the intersection of hyperplanes, rather than $\leq n-8$.

If $\left\|B_{M}\right\| \not \equiv 0$ on reg $M$, then there exists a ball $B_{r}(x)$ for some $x \in$ reg $M \cap \Omega$ and some $r>0$ such that $B_{r}(x) \cap M \subset$ reg $M$ and such that

$$
\beta \equiv \int_{B_{r}(x) \cap M}\left\|B_{M}\right\|^{2} d H^{n-1}(x)>0 .
$$

We then choose a function $\zeta$ as in Lemma 2.4 so that $\zeta=1$ on $M \cap B_{r}(x)$ and so that

$$
\int_{M}\left|\nabla_{M} \zeta\right|^{2} d H^{n-1}(x)<\beta .
$$

Consequently, (2.32) will follow from (2.31).

The verification of (2.31) results from the chain rule once we compute the quantities $\left.\frac{d^{2} V}{d t^{2}}\right|_{t=0}$ and $\left.\frac{d^{2} A}{d t^{2}}\right|_{t=0}$. Now

$$
\left.\frac{d^{2} V}{d t^{2}}\right|_{t=0}=\left.\int_{E} \frac{\partial^{2} J \Psi_{t}(x)}{\partial t^{2}}\right|_{t=0} d x .
$$


In light of (2.10) and (2.12) we find

$$
\left.\frac{\partial^{2} J \Psi_{t}}{\partial t^{2}}\right|_{t=0}=\operatorname{div} Z+(\operatorname{div} X)^{2}-X_{x_{j}}^{(i)} X_{x_{i}}^{(j)} .
$$

Hence,

$$
\left.\frac{d^{2} V}{d t^{2}}\right|_{t=0}=\int_{E} \operatorname{div} Z+(\operatorname{div} X)^{2}-X_{x_{j}}^{(i)} X_{x_{i}}^{(j)} d x
$$

But since

$$
\operatorname{div} Z=X_{x_{i} x_{j}}^{(i)} X^{(j)}+X_{x_{j}}^{(i)} X_{x_{i}}^{(j)}
$$

one can use the fact that

$$
\operatorname{div}((\operatorname{div} X) X)=(\operatorname{div} X)^{2}+X_{x_{i} x_{j}}^{(i)} X^{(j)}
$$

to conclude that

$$
\left.\frac{d^{2} V}{d t^{2}}\right|_{t=0}=\int_{E} \operatorname{div}((\operatorname{div} X) X) d x .
$$

Again invoking properties (2.2)-(2.5) we obtain

$$
\begin{aligned}
\left.\frac{d^{2} V}{d t^{2}}\right|_{t=0}= & \int_{M}(\operatorname{div} X) X \cdot \nu_{M} d H^{n-1}(x) \\
= & \int_{M} \zeta \operatorname{div}\left(\bar{\zeta} \nu_{M}\right) d H^{n-1}(x) \\
= & (n-1) H \int_{M} \zeta^{2} d H^{n-1}(x) \\
& +\int_{M} \zeta \nabla \zeta \cdot \nu_{M} d H^{n-1}(x),
\end{aligned}
$$

where $H$ is the (constant) mean curvature of reg $M$.

We now turn to the calculation of $\left.\frac{d^{2} A}{d t^{2}}\right|_{t=0}$. Recalling the notation from the proof of Proposition 2.2 in which $\Phi_{t}$ denotes the restriction of $\Psi_{t}$ to $M$, we use (2.16) to calculate

$$
\begin{aligned}
\left.\frac{d^{2} A}{d t^{2}}\right|_{t=0}= & \left.\int_{M} \frac{\partial^{2} J \Phi_{t}(x)}{\partial t^{2}}\right|_{t=0} d x \\
= & \int_{M \cap \Omega}\left(\operatorname{div}_{M} Z+\left(\operatorname{div}_{M} X\right)^{2}+\sum_{i=1}^{n-1}\left|\left(D_{\tau_{i}} X\right)^{\perp}\right|^{2}\right. \\
& \left.-\sum_{i, j=1}^{n-1}\left(\tau_{i} \cdot D_{\tau_{j}} X\right)\left(\tau_{j} \cdot D_{\tau_{i}} X\right)\right) d H^{n-1}(x)
\end{aligned}
$$


On the connectivity of boundaries of sets of minimizing perimeter 213

We will analyze each term in the last integral. With (2.19), we can evaluate the second term in the integral:

$$
\int_{M}\left(\operatorname{div}_{M} X\right)^{2} d H^{n-1}(x)=(n-1)^{2} H^{2} \int_{M} \zeta^{2} d H^{n-1}(x) .
$$

To simplify the third term in the integral appearing in (2.37), note that on $M \cap \operatorname{spt} X$ we have

$$
\left|\left(D_{\tau_{i}} X\right)^{\perp}\right|=\left|\left(D_{\tau_{i}}\left(\zeta \nu_{M}\right)\right)^{\perp}\right|=\left|D_{\tau_{i}} \zeta\right|
$$

since $\left(D_{\tau_{i}} \nu_{M}\right) \cdot \nu_{M}=0$. Hence,

$$
\int_{M} \sum_{i=1}^{n-1}\left|\left(D_{\tau_{i}} X\right)^{\perp}\right|^{2}=\int_{M}\left|\nabla_{M} \zeta\right|^{2} d H^{n-1}(x) .
$$

The fourth term in the integral in (2.37) reduces to

$$
\begin{aligned}
\int_{M} \sum_{i, j=1}^{n-1}\left(\tau_{i} \cdot D_{\tau_{j}} X\right)\left(\tau_{j} \cdot\right. & \left.D_{\tau_{i}} X\right) d H^{n-1}(x) \\
& =\int_{M} \zeta^{2}\left\|B_{M}\right\|^{2} d H^{n-1}(x)
\end{aligned}
$$

To complete the evaluation of (2.37) we must analyze the integral

$$
\int_{M} \operatorname{div}_{M} Z d H^{n-1}(x)
$$

To this end, write $\operatorname{div}_{M} Z=\operatorname{div}_{M} Z^{T}+\operatorname{div}_{M} Z^{\perp}$ where

$$
Z^{T} \equiv \sum_{i=1}^{n-1}\left(Z \cdot \tau_{i}\right) \tau_{i} \quad \text { and } \quad Z^{\perp} \equiv\left(Z \cdot \nu_{M}\right) \nu_{M} .
$$

Now on $M \cap \operatorname{spt} Z$ we have

$$
\begin{aligned}
\operatorname{div}_{M} Z^{\perp} & =\left[D_{\tau_{i}}\left(\left(Z \cdot \nu_{M}\right) \nu_{M}\right)\right] \cdot \tau_{i} \\
& =\left(Z \cdot \nu_{M}\right)\left(D_{\tau_{i}} \nu_{M}\right) \cdot \tau_{i} \\
& =(n-1) H\left(Z \cdot \nu_{M}\right) .
\end{aligned}
$$


Since (2.3) and (2.5) imply that on $M \cap$ spt $Z$ we have $Y_{x_{j}}^{(i)} Y^{(i)}=0$ for $j=$ $1, \ldots, n$ as well as $Y^{(i)}=\nu_{M}^{(i)}$, we can use (2.2)-(2.5),(2.6),(2.11) and (2.41) to calculate

$$
\begin{aligned}
\int_{M} \operatorname{div}_{M} Z^{\perp} d H^{n-1}(x) & =(n-1) H \int_{M} X_{x_{j}}^{(i)} X^{(j)} \nu_{M}^{(i)} d H^{n-1}(x) \\
& =(n-1) H \int_{M}\left(\zeta Y^{(i)}\right)_{x_{j}} \zeta Y^{(j)} \nu_{M}^{(i)} d H^{n-1}(x) \\
& =(n-1) H \int_{M} \zeta \nabla \zeta \cdot \nu_{M} d H^{n-1}(x) .
\end{aligned}
$$

We now apply the Divergence Theorem (see e.g. [S], Section 7) and the orthogonality of $M$ and $\partial \Omega$ (cf. Theorem 2.1) to obtain

$$
\int_{M} \operatorname{div}_{M} Z^{T} d H^{n-1}(x)=\int_{M \cap \partial \Omega} Z^{T} \cdot \nu_{\partial \Omega} d H^{n-2}(x) .
$$

Letting $\left\{\tau_{i}^{\prime}(x)\right\}_{i=1}^{n-2}$ be an orthonormal basis for $T_{x}(\partial \Omega) \cap T_{x} M$ for $x \in$ reg $M \cap \partial \Omega$ we observe that $\left\{\tau_{1}^{\prime}, \ldots, \tau_{n-2}^{\prime}, \nu_{\partial \Omega}\right\}$ forms an orthonormal basis for $T_{x} M$ for any $x \in \operatorname{reg} M \cap \partial \Omega$. Thus, we may write

$$
Z^{T}=\sum_{i=1}^{n-2}\left(Z \cdot \tau_{i}^{\prime}\right) \tau_{i}^{\prime}+\left(Z \cdot \nu_{\partial \Omega}\right) \nu_{\partial \Omega}
$$

so that by (2.43) and (2.11) we have

$$
\begin{aligned}
\int_{M} \operatorname{div}_{M} Z^{T} d H^{n-1}(x) & =\int_{M \cap \partial \Omega} Z \cdot \nu_{\partial \Omega} d H^{n-2}(x) \\
& =\int_{M \cap \partial \Omega} X_{x_{j}}^{(i)} X^{(j)} \nu_{\partial \Omega}^{(i)} d H^{n-2}(x) .
\end{aligned}
$$

We then invoke the condition $X \cdot \nu_{\partial \Omega}=0$ for $x \in M \cap \partial \Omega$ (cf. (2.4)) to assert that

$$
\frac{\partial}{\partial x_{j}}(X \cdot N) X^{(j)}=0
$$

where $N$ is any smooth extension of the vector field $\nu_{\partial \Omega}$. Consequently,

$$
\begin{aligned}
\int_{M} \operatorname{div}_{M} Z^{T} d H^{n-1}(x) & =-\int_{M \cap \partial \Omega} X^{(i)} N_{x_{j}}^{i} X^{(j)} d H^{n-2}(x) \\
& =-\int_{M \cap \partial \Omega} \zeta^{2} Y^{(i)} N_{x_{j}}^{(i)} Y^{(j)} d H^{n-2}(x) \\
& =-\int_{M \cap \partial \Omega} \zeta^{2} D_{\nu_{M}}\left(\nu_{\partial \Omega}\right) \cdot \nu_{M} d H^{n-2}(x)
\end{aligned}
$$


Applying (2.38), (2.39), (2.40), (2.42) and (2.45) to the formula (2.37) we conclude that

$$
\left.\frac{d^{2} A}{d t^{2}}\right|_{t=0}=(n-1)^{2} H^{2} \int_{M} \zeta^{2} d H^{n-1}(x)+\int_{M}\left|\nabla_{M} \zeta\right|^{2} d H^{n-1}(x)
$$

$$
\begin{aligned}
& -\int_{M} \zeta^{2}\left\|B_{M}\right\|^{2} d H^{n-1}(x)+(n-1) H \int_{M} \zeta \nabla \zeta \cdot \nu_{M} d H^{n-1}(x) \\
& -\int_{M \cap \partial \Omega} \zeta^{2} D_{\nu_{M}}\left(\nu_{\partial \Omega}\right) \cdot \nu_{M} d H^{n-2}(x) .
\end{aligned}
$$

Now from the chain rule we have

$$
\frac{d^{2} \mathcal{A}}{d V^{2}}=\frac{\frac{d V}{d t} \frac{d^{2} A}{d t^{2}}-\frac{d A}{d t} \frac{d^{2} V}{d t^{2}}}{\left(\frac{d V}{d t}\right)^{3}} .
$$

A substitution of (2.14), (2.19), (2.36) and (2.46) into (2.47) yields (2.31).

As an application of Theorem 2.5, we obtain:

Theorem 2.6. Let $\Omega \subset \mathbb{R}^{n}$ be a bounded, convex open set with $C^{2, \alpha}$ boundary. Let $E$ be a local minimizer of perimeter with respect to fixed volume in the sense of (2.1) and let $M \equiv \overline{\Omega \cap \partial E}$. Then either $M$ is connected or it consists of a union of parallel planar components meeting $\partial \Omega$ orthogonally with that part of $\Omega$ lying between any two such planar components consisting of a cylinder. In particular, if $\Omega$ is strictly convex in the sense that $B_{\partial \Omega}(\tau(x), \tau(x))>0$ for all $x \in \partial \Omega$ and $\tau(x) \in T_{x}(\partial \Omega)$, then $M$ is connected.

Proof. Suppose by way of contradiction that $\overline{\Omega \cap \partial E}$ consists of more than one component. Denote any two of these components by $M_{1}$ and $M_{2}$. In light of Theorem 2.1 we know that $\overline{\Omega \cap \partial E}$ is regular off of a singular set of Hausdorff dimension at most $n-8$. Furthermore, $M_{1}$ and $M_{2}$ are separated from each other and from any other components of $\overline{\Omega \cap \partial E}$ by some positive distance. Suppose first that either

$$
\left\|B_{M_{1}}\right\| \not \equiv 0 \quad \text { or } \quad\left\|B_{M_{2}}\right\| \not \equiv 0 .
$$

Say $\left\|B_{M_{1}}\right\| \not \equiv 0$. Then by Theorem 2.5 , there exist functions $\zeta^{(1)}$ and $\zeta^{(2)}$ supported in neighborhoods of $M_{1}$ and $M_{2}$ respectively and two associated 
diffeomorphisms of $\mathbb{R}^{n},\left(\Psi_{i}\right)_{t}, i=1,2$ with

$$
\operatorname{spt}\left(\left(\Psi_{1}\right)_{t}-I\right) \cap \operatorname{spt}\left(\left(\Psi_{2}\right)_{t}-I\right)=\emptyset
$$

such that

$$
\left.\frac{d^{2} \mathcal{A}_{1}}{d V_{1}^{2}}\right|_{V_{1}=|E|}<0 \quad \text { while }\left.\quad \frac{d^{2} \mathcal{A}_{2}}{d V_{2}^{2}}\right|_{V_{2}=|E|} \leq 0 .
$$

Here $V_{i}(t)=\left|\left(\Psi_{i}\right)_{t}(E)\right|$ for $i=1,2$, and we have denoted by $\mathcal{A}_{i}\left(V_{i}\right), i=1,2$, the relation between perimeter $P\left(\left(\Psi_{i}\right)_{t}(E), \Omega\right)$ and volume $V_{i}(t)$ for each variation.

As both $V_{1}$ and $V_{2}$ are monotone functions of $t$ for $|t|$ small, while $V_{1}(0)=$ $V_{2}(0)=|E|$, we may find for each small $t$ a unique number $s(t)$ such that $\left(V_{1}(t)-|E|\right)+\left(V_{2}(s(t))-|E|\right)=0$ or simply

$$
V_{1}(t)+V_{2}(s(t))=2|E| .
$$

Now define the family of competing sets $E_{t}$ via the formula

$$
E_{t} \equiv \Psi_{t}(E) \quad \text { where } \quad \Psi_{t}(x)=\left(\Psi_{1}\right)_{t}(x)+\left(\Psi_{2}\right)_{s(t)}(x)-x \quad \text { for all } x \in \mathbb{R}^{n} .
$$

Note that $\Psi_{t}-I$ is only supported in a neighborhood of $M_{1}$ and $M_{2}$ and that $\left|E_{t}\right|=|E|$ in light of (2.50).

In light of (2.50), we may express the total perimeter within $\Omega$ of the set $E_{t}$ as a function of $V_{1}$ alone, and we denote this relationship by $\mathcal{A}\left(V_{1}\right)$. It follows from Proposition 2.2 and (2.50) that

$$
\begin{aligned}
\left.\frac{d \mathcal{A}}{d V_{1}}\right|_{V_{1}=|E|} & =\left.\frac{d \mathcal{A}_{1}}{d V_{1}}\right|_{V_{1}=|E|}+\left.\frac{d \mathcal{A}_{2}}{d V_{1}}\right|_{V_{1}=|E|} \\
& =\left.\frac{d \mathcal{A}_{1}}{d V_{1}}\right|_{V_{1}=|E|}-\left.\frac{d \mathcal{A}_{2}}{d V_{2}}\right|_{V_{2}=|E|} \\
& =(n-1) H-(n-1) H=0 .
\end{aligned}
$$

However, (2.49) and (2.50) imply

$$
\begin{aligned}
\left.\frac{d^{2} \mathcal{A}}{d V_{1}^{2}}\right|_{V_{1}=|E|} & =\left.\frac{d^{2} \mathcal{A}_{1}}{d V_{1}^{2}}\right|_{V_{1}=|E|}+\left.\frac{d^{2} \mathcal{A}_{2}}{d V_{1}^{2}}\right|_{V_{1}=|E|} \\
& =\left.\frac{d^{2} \mathcal{A}_{1}}{d V_{1}^{2}}\right|_{V_{1}=|E|}+\left.\frac{d^{2} \mathcal{A}_{2}}{d V_{2}^{2}}\right|_{V_{2}=|E|}<0 .
\end{aligned}
$$


Hence, for small values of $t$ we conclude that $P\left(E_{t}, \Omega\right)<P(E, \Omega)$ while $\left|E_{t}\right|=|E|$, contradicting the local minimality of $E$.

It remains to treat to the case where (2.48) fails. If $\left\|B_{M_{i}}\right\| \equiv 0$ for both $i=1$ and 2 and $\Omega$ is strictly convex, then formula (2.49) still holds by Theorem 2.5 and the contradiction is again reached.

Finally, suppose both $M_{1}$ and $M_{2}$ are planar and meet $\partial \Omega$ orthogonally only at points where

$$
B_{\partial \Omega}\left(\nu_{M_{i}}, \nu_{M_{i}}\right)=0 .
$$

Let $\Pi_{1}$ and $\Pi_{2}$ denote the two planes containing $M_{1}$ and $M_{2}$ respectively. We first claim that $\Pi_{1}$ and $\Pi_{2}$ must be parallel. To see this, let $x \in M_{1}$ and $y \in M_{2}$ achieve the minimal distance between the two components. If both $x$ and $y$ lie in $\Omega$ then clearly the planes are parallel, so without loss of generality, we may assume $x \in \partial \Omega$. Now suppose, by way of contradiction, that the line segment $\overline{x y}$ is not orthogonal to $M_{1}$. Then $x$ is not the closest point of $\Pi_{1}$ to $y$ and indeed such a closest point, say $z$, must lie outside $\bar{\Omega}$. Consider the triangle formed by $x, y$ and $z$. The angle $\angle x z y$ must be $90^{\circ}$ since $\overline{y z} \perp \Pi_{1}$. However, the convexity of $\Omega$ forces $y$ and $z$ to lie on opposite sides of $T_{x}(\partial \Omega)$, and since $\overline{x z} \perp \partial \Omega$, the angle $\angle y x z$ must exceed $90^{\circ}$ and we reach a contradiction. We conclude that $\overline{x y} \perp M_{1}$. Similarly, $\overline{x y} \perp M_{2}$ and the two planes are parallel. But convexity requires $\overline{x y} \subset \bar{\Omega}$ and since $\nu_{M_{1}}(x) \in T_{x}(\partial \Omega)$ implies $x+t \nu_{M_{1}}(x) \notin \Omega$ for all $t \in \mathbb{R}^{1}$, it follows that $\overline{x y} \subset \partial \Omega$. Consequently, that part of $\Omega$ lying between the parallel planes $\Pi_{1}$ and $\Pi_{2}$ must be the cylinder $M_{1} \times(0,|x-y|)$.

Finally, we present some consequences of Theorems 2.5 and 2.6 related to absolute minimizers of perimeter subject to a volume constraint.

Lemma 2.7. Let $f: I \rightarrow \mathbb{R}^{1}$ be a lower-semicontinuous function defined on an interval $I$ and suppose $f$ is locally concave in the sense that its graph admits a local upper support line in a neighborhood of any point on the graph. Then, $f$ is concave.

Proof. Equivalently, we must show that the graph of $f$ lies completely above any secant line. Assume to the contrary that it extends below a secant between $s=a$ and $s=b$, which can be taken without loss of generality to be horizontal. By lower-semicontinuity, a minimum is achieved over the interval $[a, b]$ and necessarily, it occurs in $(a, b)$. Furthermore, the set of $s$-values where this minimum is achieved is closed. By the local support 
line property, however, this set of $s$-values is also open. Hence, $f$ must be constant on $[a, b]$, a contradiction.

Remark. The assumption of semicontinuity is clearly necessary. For example, the function $f(s)$ defined as $|s|$ for $s \neq 0$ and 1 for $s=0$ is locally concave but not concave.

Theorem 2.8. Let $\Omega \subset \mathbb{R}^{n}$ be an open, bounded, strictly convex set with $C^{2, \alpha}$ boundary. Then the function

$$
P(m) \equiv \inf _{F \in \mathcal{A}_{m}} P(F, \Omega),
$$

is a concave function of $m$ on the interval $0 \leq m \leq|\Omega|$ and $P$ is symmetric about $|\Omega| / 2$.

Proof. One can easily verify that $P$ is a continuous function of $m$ and the symmetry in $m$ is immediate by taking complements within $\Omega$. To check the concavity, fix any volume $m_{0}$ in the interval $(0,|\Omega|)$. Let $E$ be an absolute minimizer for that volume. By Theorem 2.6, we know $M \equiv \overline{\Omega \cap \partial E}$ is connected. Denote the constant mean curvature of reg $M$ by $H$. For this $M$, we apply Proposition 2.2 and Theorem 2.5 in order to construct a family of sets $\left\{E_{t}\right\}$ with $E_{0}=E$ such that the function $\mathcal{A}(m)$ measuring $P\left(E_{t}, \Omega\right)$ as a function of $m=\left|E_{t}\right|$ is concave and satisfies $\left.\frac{d \mathcal{A}}{d m}\right|_{m=m_{0}}=(n-1) H$. This function may only be defined for $m$ in some small interval about $m_{0}$, but within its domain of definition, it necessarily satisfies $\mathcal{A}(m) \geq P(m)$ and $\mathcal{A}\left(m_{0}\right)=P\left(m_{0}\right)$. In particular, the line

$$
l(m)=(n-1) H\left(m-m_{0}\right)+P\left(m_{0}\right)
$$

is locally an upper support line for both $\mathcal{A}(m)$ and $P(m)$. Since $P$ is also continuous, the previous lemma implies that $P$ is a concave function of $m$.

Corollary 2.9. Let $\Omega \subset \mathbb{R}^{n}$ be an open, bounded, strictly convex set with $C^{2, \alpha}$ boundary. Then, for $m<|\Omega| / 2$, any absolute minimizer of

$$
\inf _{F \in \mathcal{A}_{m}} P(F, \Omega)
$$

must have strictly positive mean curvature. 
Proof. The mean curvature of any absolute minimizer must be in the superdifferential of the function $P(m)$ of Theorem 2.8; otherwise, the line $l$ of (2.53) would not be a local upper support line. (By the superdifferential at a point $x$, we mean the set of slopes of all upper support lines to the graph of $P$ at $x$.) By concavity, the superdifferential is decreasing in the sense that, for $x>y$, every value in the superdifferential at $x$ lies below every value in the superdifferential at $y$. But, by symmetry, zero is in the superdifferential at $|\Omega| / 2$. It follows that each value in the superdifferential is positive for $x<|\Omega| / 2$.

The property expressed in Corollary 2.9, though quite intuitive, captures global information. It does not hold for local minimizers. For example, take $\Omega$ to be the unit disc in the plane. Then the annulus of inner radius $3 / 4$ and outer radius 1 is a local minimizer with respect to its own measure but has a negatively curved boundary. Alternatively, consider for example, an absolute minimizer $E$ in a convex domain $\Omega \subset \mathbb{R}^{n}$ such that $|E|$ is just below $|\Omega| / 2$. By trimming $\Omega$ far away from $E$, one can create a new convex domain $\Omega^{\prime} \subset \Omega$ such that $E$ is a local minimizer within $\Omega^{\prime}$ with positive mean curvature and volume satisfying $|E|>|\Omega| / 2$.

We also remark that the function $P(m)$, though continuous, is typically not $C^{1}$. Jumps in the derivative occur wherever there exist multiple absolute minimizers with different mean curvatures. Generically, such jumps occur at $m=|\Omega| / 2$, since typically an absolute minimizer $E$ for this $m$-value will have mean curvature $H \neq 0$ and both $E$ and $\Omega \backslash E$ will solve the problem. To construct examples with jumps at values other than $m=|\Omega| / 2$, one can round off the corners of a triangular domain using varying radii of curvature. For some value of $m$, there will then be absolute minimizers near two of the (former) corners, with different radii of curvature but enclosing the same volume.

Finally, we do not expect that absolute minimizers need be convex, since nonplanar minimal surfaces always have principal curvatures of opposing signs and the same is often true of constant mean curvature hypersurfaces. It should be possible to construct a nonconvex minimizer, for example, by perturbation of a strictly stable, planar minimizer. 
Acknowledgments. We thank Bruce Solomon and William Ziemer for numerous fruitful discussions related to this work.

\section{References.}

[G] E. Giusti, Minimal surfaces and functions of bounded variation, Birkhäuser, 1984.

[Gr] M. Grüter, Boundary regularity for solutions of a partitioning problem, Arch. Rat. Mech. Anal. 97 (1987), 261-270.

[GJ] M. Grüter and J. Jost, Allard type regularity results for varifolds with free boundaries, Ann. Sc. Norm. Sup. di Pisa 13 (1986), 129-169.

[GMT] E. Gonzalez, U. Massari and I. Tamanini, On the regularity of boundaries of sets minimizing perimeter with a volume constraint, Indiana Univ. Math. J. 32 (1983), 25-37.

[KS] R. Kohn and P. Sternberg, Local minimizers and singular perturbations, Proc. Roy. Soc. Edin. 111A (1989), 69-84.

[S] L. Simon, Lectures on Geometric Measure Theory, Proc. Centre for Math. Anal., Australian Nat. Univ., 3 (1983).

[SZ] P. Sternberg and K. Zumbrun, Connectivity of phase boundaries in strictly convex domains, Arch. Rat. Mech. Anal., 141(1998), 375-400.

Received August 20, 1998.

Department of Mathematics

INDIANA UNIVERSITY

BLOOMINGTON, IN 47405 